\title{
Deformation Enhanced Diffusion in Aluminium Alloys
}

\begin{abstract}
J.D. ROBSON
Deformation introduces defects such as dislocations and excess vacancies that can strongly influence diffusion rates in aluminium alloys. This is of great importance in understanding the effect of deformation on processes such as precipitation hardening, which can be accelerated by orders of magnitude by the defects introduced. In this work, a simple and widely used classical model has been employed to explore the effect of process variables on diffusion enhancement due to deformation-induced excess vacancies and dislocations. It is demonstrated that in aluminium alloys, the strain rates and temperatures used in processing and testing encompass a range of regimes. At low strain rate or high temperature, a steady state becomes established in which the deformation enhancement depends on strain rate but not strain. Conversely, at high strain rate or low temperature, deformation enhancement is insensitive to strain rate but increases with strain. For all conditions, the effect of excess vacancies is much stronger than the direct effect of pipe diffusion along dislocations. The predicted deformation-induced vacancy concentration can exceed that expected after rapid quenching by an order of magnitude, but the lifetime of the deformation-induced vacancies is much shorter. Finally, limitations of the classical model and suggestions for improvement are proposed.
\end{abstract}

https://doi.org/10.1007/s11661-020-05960-5

(C) The Author(s) 2020

\section{INTRODUCTION}

High-STRENGTH aluminium alloys rely on decomposition of a supersaturated solid solution to form precipitates that act as obstacles to dislocation motion. This process requires movement and aggregation of solute atoms, which is achieved during a heat treatment process by thermally activated diffusion. The scenario is made considerably more complex when precipitation occurs simultaneously with plastic deformation. In this case, solute transport can be accelerated either directly or indirectly by the dislocation motion that produces plasticity. This is of great practical importance in processes such as warm forming, creep age forming, high pressure torsion, and fatigue, where strong dynamic interactions can occur. ${ }^{[1-6]}$

The nature of the interaction between precipitate evolution and deformation depends both on the initial microstructural state of the material (e.g. degree of supersaturation) and on the deformation conditions (temperature, strain rate, etc.). A full discussion of possible interactions is detailed elsewhere, ${ }^{[1,3,7]}$ but important effects for aluminium alloys include direct precipitation on dislocations (dynamic strain ageing $\left.^{[1,8,9]}\right)$, ballistic transfer of solute by slip, ${ }^{[1]}$ and

J.D. Robson is with the Department of Materials, University of Manchester, Manchester M13 9PL. Contact e-mail: joseph.robson@manchester.ac.uk

Manuscript submitted on June 3, 2020.

Article published online August 18, 2020 deformation-induced vacancies . Recent in situ studies suggest a dominant role for strain-induced excess vacancies in enhancing diffusivity, at least at temperatures less than approximately $473 \mathrm{~K}\left(200^{\circ} \mathrm{C}\right) .^{[2,3,5,10]}$ The idea that part of the work applied during straining goes into the generation of excess vacancies is well established. ${ }^{[11,12]}$ By estimating the fraction of applied work consumed in this process, a determination can be made of the excess vacancy concentration that is generated. ${ }^{[11,12]}$ Excess vacancies will strongly influence diffusivity and other kinetic processes governing precipitation. ${ }^{[3]}$

The deformation-induced vacancies can arise from a number of sources, ${ }^{[13,14]}$ but the dominant one is considered to be non-conservative motion of conventional jogs on screw dislocations. Low-temperature deformation and annealing studies suggest that the deformation-induced excess vacancy concentrations approach $10^{-4},{ }^{[15,16]}$ orders of magnitude greater than the equilibrium thermal vacancy concentration and close to that expected near the melting point. Since the diffusivity of substitutional solute species is directly proportional to the vacancy concentration, this huge increase in the number of vacancies will result in a huge effect on self and solute diffusion rates.

Simple models are available that attempt to capture dynamic effects on precipitate evolution. One of the most widely used models for the excess vacancy effect is due to Militzer et al. ${ }^{[17]}$ Although not expected to be highly qualitatively accurate, such models are invaluable in helping to understand the fundamental dependencies 
between process conditions and dynamic effects in the microstructure. ${ }^{[2,3,18]}$

Models can help explain why different regimes of behaviour are observed depending on conditions. For example, increasing strain rate has been shown to have either no effect on dynamic enhancement (for a given level of strain $)^{[2,3]}$ or a negative effect. ${ }^{[1,3,10]}$ As will be demonstrated later, in aluminium alloys, both regimes can be produced by differences in temperature, strain, and strain rate that fall within practical ranges. This has important consequences for industrial processing, particularly of age hardenable aluminium alloys. There is also an opportunity to exploit a better understanding of dynamic interactions to accelerate ageing or optimize precipitate distribution. ${ }^{[5]}$

Since the problem of coupled precipitation and deformation is complex, this paper will focus only on the effect of deformation on diffusivity and the problem of precipitate nucleation will not be considered. ${ }^{[3]}$ Diffusivity enhancement is the dominant effect of deformation when a pre-existing precipitate population has been nucleated and is in the growth and coarsening regimes. In an industrial forming setting, it is envisaged that a pre-ageing process will be performed prior to deformation (forming), and therefore the diffusivity effect will dominate subsequent precipitate evolution.

The purpose of this paper is to explore the consequences of a simple model that predicts the deformation-induced excess vacancy concentration for conditions typical of those encountered in mechanical testing and thermomechanical processing of aluminium alloys. The significance of excess vacancies will be compared with other effects that can enhance diffusion rates. The sensitivity of the model to input parameters is explored, and suggestions for improvement are discussed.

\section{MODEL}

Two deformation-induced defect types that lead to enhance diffusion are considered in the model: dislocations and deformation-induced vacancies. For deformation-induced vacancies, the widely used model of Militzer et al. was used. ${ }^{[17]}$ This considers the balance between production of excess vacancies by dislocation motion and their annihilation by diffusion to sinks. The resultant rate of excess vacancy production is given by:

$$
\frac{\mathrm{d} c_{\mathrm{ex}}}{\mathrm{d} t}=\left(\chi \frac{\sigma \Omega_{0}}{Q_{\mathrm{f}}} \dot{\epsilon}+\zeta \frac{c_{j} \Omega_{0}}{4 b^{3}} \dot{\epsilon}\right)-\left(\frac{D_{\mathrm{v}} \rho}{\kappa^{2}} c_{\mathrm{ex}}+\frac{D_{\mathrm{v}}}{L^{2}} c_{\mathrm{ex}}\right) .
$$

The first set of parentheses contain two terms corresponding to the mechanical and thermal contributions to vacancy production respectively. The mechanical term is a phenomenological expression of the observation that a fraction of the applied work during deformation is converted into excess vacancies. A constant $\chi$ expresses this fraction, and is usually set to 0.1 . The implications of this assumption are discussed later. The contribution from thermal jogs is only important above about 0.4 of the melting temperature ${ }^{[17]}$ (i.e. $T>373 \mathrm{~K}$ $\left(100^{\circ} \mathrm{C}\right)$ for aluminium). The calculation of the thermal jog contribution requires calculating the concentration of thermal jogs, which depends on the thermal jog formation energy $E_{j}$. The procedure used by Militzer et al. was used to calculate these parameters, details elsewhere. ${ }^{[17]}$

The second set of parentheses contains two terms corresponding to annihilation of excess vacancies at dislocations and grain boundaries respectively. This depends on the dislocation density $\rho$, dislocation arrangement $\kappa$, vacancy diffusion coefficient $D_{\mathrm{v}}$, and grain size $L$. The concentration of thermal jogs and the vacancy diffusion coefficient are calculated as a function of temperature using the usual Arrhenius expressions. ${ }^{[17]}$ For example, the vacancy diffusion coefficient is given by

$$
D_{\mathrm{v}}=D_{\mathrm{v} 0} \exp \left(-\frac{Q_{\mathrm{m}}}{k T}\right),
$$

where $Q_{\mathrm{m}}$ is the activation energy for vacancy migration.

A simple model for the dislocation density evolution is used, following Militzer et al. ${ }^{[17]}$ The evolving dislocation density as a function of time is given by

$$
\rho(t)=\rho_{\mathrm{s}}-\left(\rho_{\mathrm{s}}-\rho(0)\right) \exp \left(-\frac{t}{\beta}\right)
$$

where $\rho_{\mathrm{s}}$ is the steady-state dislocation density during deformation, $\rho(0)$ is the initial dislocation density, and the characteristic time $\beta$ is given by

$$
\beta=\frac{\sigma}{\beta_{0} \dot{\epsilon}},
$$

where $\sigma$ is the flow stress, and $\beta_{0}$ is a material constant $(\simeq 500 \mathrm{MPa})$. Following Milizer et al., the flow stress of the material was fitted using a standard hyperbolic sine law, for which constants were used for AA7075, taken as a typical example of a high-strength aluminium alloy. ${ }^{[19]}$ Details of the constants used in the flow stress law are given elsewhere. ${ }^{[19]}$ Note that compared to the several order of magnitude effects directly attributed to changes in temperature or strain rate, the effect of errors in dislocation density prediction due to the simplistic model used here are expected to be small.

The deformation-induced vacancies and dislocations will enhance solute diffusivity, and the resultant effective diffusion coefficient can be written as a function of the thermal diffusion coefficient as

$$
D_{\mathrm{ef}}=D\left(1+\frac{c_{\mathrm{ex}}}{c_{\mathrm{th}}}+g \frac{D_{\mathrm{p}}}{D}\right),
$$

where $D$ is the thermal diffusion coefficient (without deformation), $c_{\mathrm{th}}$ is the concentration of thermal vacancies, $D_{\mathrm{p}}$ is the pipe diffusivity along dislocations and $g$ is the fraction of atoms associated with the dislocation pipe. Taking a typical assumption that the cross section of the dislocation core contains two atoms, $g$ can be written as 


$$
g=2 \Omega_{0} \frac{\rho}{b} .
$$

Since the activation energy for diffusion along the dislocation pipe (required to calculate $D_{\mathrm{p}}$ ) is not well known, it was assumed to be similar to that for grain boundary diffusion, approximately $0.55 Q_{\mathrm{s}}$, the activation energy for lattice diffusion. ${ }^{[20]}$

Using these equations, the effect of deformation on the effective diffusivity can be estimated, and the relative contribution of excess vacancies and dislocation pipes can be assessed. The effect of important process variables such as strain rate, strain, and temperature can also be explored.

\section{MODEL PREDICTIONS}

\section{A. Excess Vacancies: Effects of Temperature, Strain, and Strain Rate}

As Eq. [1] shows, the net rate of excess vacancy production depends on the competition between mechanisms producing new deformation-induced vacancies and mechanisms that destroy them. In aluminium alloys of a typical grain size (e.g. $>10 \mu \mathrm{m})$ the annihilation at grain boundaries is negligible compared to the annihilation at dislocations. Since both the excess vacancy concentration and dislocation (sink) density evolve during deformation, a number of complex behaviours can be produced depending on the conditions. As demonstrated later, the balance between the production and annihilation terms determine the sensitivity of excess vacancy concentration to strain, strain rate, and temperature. Since the annihilation rate depends on the excess vacancy concentration it will increase with strain and eventually a steady state is reached where generation and annihilation terms are equal.

A key difficulty in this model is accurately determining the input parameters, since they are both difficult to measure during deformation and dependent on local interactions at the atomic scale. One of the most influential yet ill-defined parameters is the vacancy migration activation energy $\left(Q_{\mathrm{m}}\right)$, which determines the temperature dependence of $D_{\mathrm{v}}$. A range of values for pure aluminium and aluminium alloys are reported in the literature, from $Q_{\mathrm{m}}=0.58-0.9 \mathrm{eV} \cdot{ }^{[22]}$ In alloys, the activation energy for vacancy migration can be strongly influenced by the formation of vacancy-solute clusters, which is an additional complicating factor (e.g. Reference 24). For warm deformed aluminium alloy, choosing different values in this range can produce different behaviour. For example, Figure 1 shows the effect of varying $Q_{\mathrm{m}}$ from 0.7 to $1 \mathrm{eV}$ for typical conditions for warm tensile testing of aluminium $\left(\dot{\epsilon}=10^{-3} \mathrm{~s}^{-1}\right.$, $T=473 \mathrm{~K}, 200^{\circ} \mathrm{C}$ ). The simulated deformation time is 100 seconds, after which the excess vacancy decay is tracked for a further 1 second (at the same temperature). The assumed value for dislocation density and the other input parameters are those used previously for aluminium alloys, ${ }^{[2]}$ summarized in Table I.

In Figure 1, the total vacancy concentration (excess + thermal vacancies) is plotted as a multiple of the equilibrium thermal vacancy concentration. As Figure 1 shows, the small changes to $Q_{\mathrm{m}}$ produce very large differences both in the predicted excess vacancy concentration, but also (and critically) in the variation of excess vacancy concentration with time (strain). For low $Q_{\mathrm{m}}$, the annihilation term is important and the excess

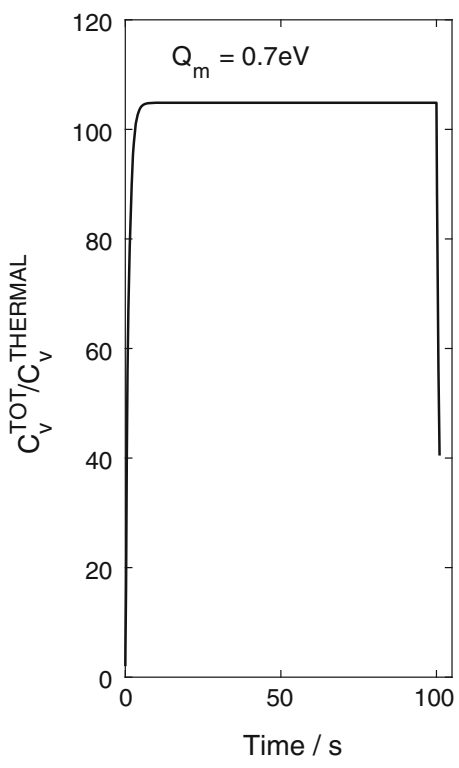

(a)

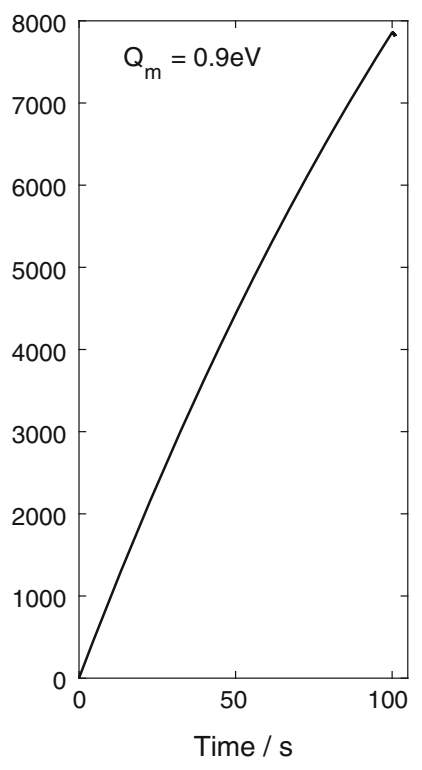

(b)

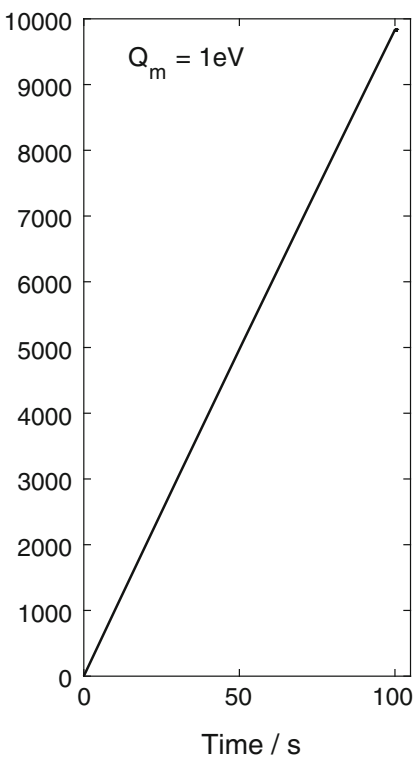

(c)

Fig. 1-Predicted effect on increasing the activation energy for vacancy migration $\left(Q_{\mathrm{m}}\right)$ on the total vacancy concentration (thermal + deformation induced) relative to the thermal vacancy concentration $\left(473 \mathrm{~K},\left(200{ }^{\circ} \mathrm{C}\right), \dot{\epsilon}=10^{-3} \mathrm{~s}^{-1}\right)(a) Q_{\mathrm{m}}=0.7 \mathrm{eV},(b) Q_{\mathrm{m}}=0.9 \mathrm{eV},(c)$ $Q_{\mathrm{m}}=1 \mathrm{eV}$. 
Table I. Model Parameters, Values Used, and Their Sources (This Work If No Citation Given)

\begin{tabular}{lll}
\hline Symbol & \multicolumn{1}{c}{ Meaning } & Value \\
\hline$\chi$ & mechanical vacancy production constant & 0.1 \\
$\Omega_{0}$ & atomic volume & $1.66 \times 10^{-29} \mathrm{~m}^{3[2]}$ \\
$Q_{f}$ & vacancy formation energy & $0.76 \mathrm{eV}^{[2]}$ \\
$E_{j}$ & jog formation energy & $0.30 \mathrm{eV}^{[17]}$ \\
$b$ & burgers vector & $0.29 \mathrm{~nm}^{[21]}$ \\
$Q_{\mathrm{m}}$ & activation energy for vacancy diffusion & $0.58 \mathrm{to}^{[21} 0.9 \mathrm{eV}^{[22]}$ \\
$D_{v 0}$ & pre-factor for vacancy diffusion & $1 \times 10^{-5} \mathrm{~m}^{2} \mathrm{~s}^{-1[22]}$ \\
$\rho$ & reference dislocation density & $2 \times 10^{12} \mathrm{~m}^{-2[2]}$ \\
$\kappa$ & dislocation arrangement & 1 to $10^{[17]}$ \\
$L$ & grain size & $50 \mu \mathrm{m}$ \\
$D_{s 0}$ & pre-factor for solute $(\mathrm{Cu})$ lattice diffusion & $2.9 \times 10^{-5} \mathrm{~m}^{-2} \mathrm{~s}^{-1[23]}$ \\
$Q_{s}$ & activation energy for solute $(\mathrm{Cu})$ lattice diffusion & $125 \mathrm{~kJ} \mathrm{~mol}^{-1[23]}$ \\
\hline
\end{tabular}

vacancy concentration rapidly reaches a steady state (within $10 \mathrm{~s}, \epsilon=1 p c t$ ). After deformation, the excess vacancies are lost rapidly, and more than half of them are annihilated after 1 second. In contrast, for the highest $Q_{\mathrm{m}}$ value, annihilation is negligible over the duration simulated, and the excess vacancy concentration increases linearly with time (strain). There is also negligible annihilation of the excess vacancies in the 1 second interval after deformation. The intermediate value of $Q_{\mathrm{m}}=0.9 \mathrm{eV}$ represents a case where the increase in excess vacancies is almost linear with time (strain), but annihilation becomes significant at longer times, so that the maximum excess vacancy concentration reached is significantly less than would be the case with no annihilation. Again, in this case, annihilation in the 1 second after deformation is negligible. When $Q_{\mathrm{m}}=$ $1 \mathrm{eV}$ annihilation is negligible during and after deformation. Therefore, increasing $Q_{\mathrm{m}}$ over the reported reasonable range, changes the predicted behaviour from a steady-state excess vacancy concentration with strain to a linearly increasing concentration.

\section{B. Activation Energy for Vacancy Annihilation}

As already demonstrated, the model predictions are sensitive to the activation energy for vacancy diffusion, but this parameter is poorly defined and furthermore is expected to be a function of alloy composition. The difficulty in measuring the activation energy for diffusion of vacancies arises from the difficulty in measuring vacancy concentrations themselves, particularly when evolving with deformation or temperature. Proxy methods, such as measuring changes in resistivity correlated to vacancy concentration, ${ }^{[13]}$ or more direct methods using positron annihilation ${ }^{[25]}$ or nuclear magnetic resonance ${ }^{[26]}$ have been used. These methods are significantly complicated by the presence of other alloying additions and defects present in alloys of practical interest. They are also too slow to measure the rapidly evolving dynamic vacancy concentration.

An alternative pragmatic method for determining the effective activation energy for vacancy diffusion was used in this work. Once the deformation is stopped, the excess vacancies decay and this decay is reflected in a

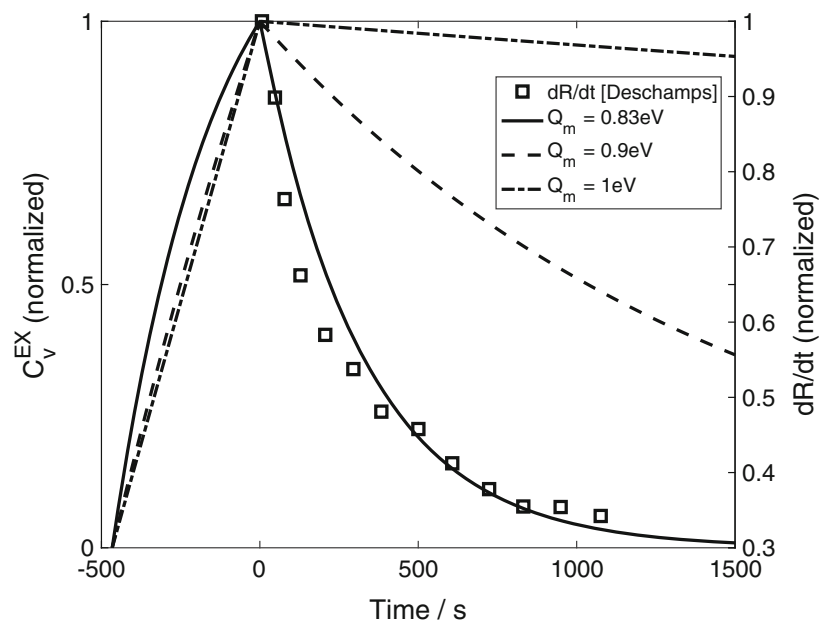

Fig. 2-Predicted decay in excess vacancy concentration after deformation for 3 vales of the activation energy for vacancy migration $Q_{\mathrm{m}}$, compared with the decay in growth rate measured in situ by Deschamps et al. ${ }^{[2]}$

reduction in the precipitate growth rate. The decay rate of excess vacancies is strongly dependent on the activation energy for vacancy diffusion. Therefore, the effective activation energy for vacancy diffusion can be found by fitting to produce the correct decay behaviour in the growth rate. As an example, Deschamps et al. ${ }^{[2]}$ have performed in situ measurements that track the decay in growth rate after deformation is completed. Using reasonable values for dislocation density and other parameters (Table I), adjusting $Q_{\mathrm{m}}$ is necessary to give the correct growth rate decay behaviour. Providing the adjusted value is within the physically reasonable range $\left(Q_{\mathrm{m}}=0.65-0.9 \mathrm{eV}\right)$ this provides an indirect method for determining the correct value to use to properly capture the excess vacancy annihilation rate. As an example, Figure 2 shows the effect of adjusting $Q_{\mathrm{m}}$ on the predicted decay behaviour compared with the decay in growth rate measured by Deschamps et al. (at $433 \mathrm{~K}$ $\left.\left(160{ }^{\circ} \mathrm{C}\right) \dot{\epsilon} \simeq 10^{-5} \mathrm{~s}^{-1}\right)$. It can be seen that $Q_{\mathrm{m}}$ values at the top of the plausible range give decay behaviour which is too slow compared with the measured decay in 
the growth rate. A fitted $Q_{\mathrm{m}}$ value of $0.83 \mathrm{eV}$ gives a good fit to the measured decay behaviour.

\section{Application of Model: Constant Dislocation Density}

Equation [1] can be applied under different conditions of strain rate and temperature to understand the likely accelerating effect of excess vacancies formed during deformation on diffusion. In practice, the sources and sinks of excess vacancies (dislocations) also evolve during the deformation process. However, at first, this will be ignored and a steady-state and temperature-independent dislocation density will be assumed. This allows the predicted effect of process variables on the excess vacancy concentration to be isolated without the complication of an evolving dislocation density, which will be considered later.

Figure 3 shows the effect of large changes in either strain rate $(a-b)$ or temperature (c-d). In all cases, the total simulated strain is 10 pct. For a slow strain rate, typical of a laboratory tensile test at a temperature of $473 \mathrm{~K}\left(200{ }^{\circ} \mathrm{C}\right)$, the excess vacancy concentration is predicted to increase throughout the test, but the curvature of the plot is evidence of the significance of vacancy annihilation. At 100 seconds, vacancy generation was turned off (no further deformation), indicated by the vertical line in the plots. As shown in (a),
10 seconds after the deformation at $473 \mathrm{~K}\left(200^{\circ} \mathrm{C}\right)$, it is predicted that most of the excess vacancies still remain. If the strain rate is increased to 1 at the same temperature (b), then vacancy annihilation becomes negligible, and the excess vacancy concentration increases in proportion to the applied strain, reaching a value that is over 3 times greater than at the slow strain rate. Retaining a strain rate of 1 , but reducing the temperature to $373 \mathrm{~K}\left(100{ }^{\circ} \mathrm{C}\right)$ gives an even greater predicted excess vacancy concentration, with no significant annihilation during deformation, and a long decay time for the excess vacancies generated (this point will be explored later in more detail). Finally, on increasing the temperature to $673 \mathrm{~K}\left(400{ }^{\circ} \mathrm{C}\right)$, vacancy annihilation becomes much more rapid, meaning that the excess vacancy concentration is always orders of magnitude less than at the other temperatures simulated. In this case the excess vacancy concentration decays rapidly (within about 0.5 second after deformation is turned off).

As will be explored later, the approximations and limitations of the excess vacancy model as expressed in Eq. [1] mean that the predictions are not expected to be qualitatively accurate. However, the qualitative trends evident in Figure 3 are expected to be reasonable. In particular, the very strong effect of temperature on vacancy annihilation rate means that the effect of excess

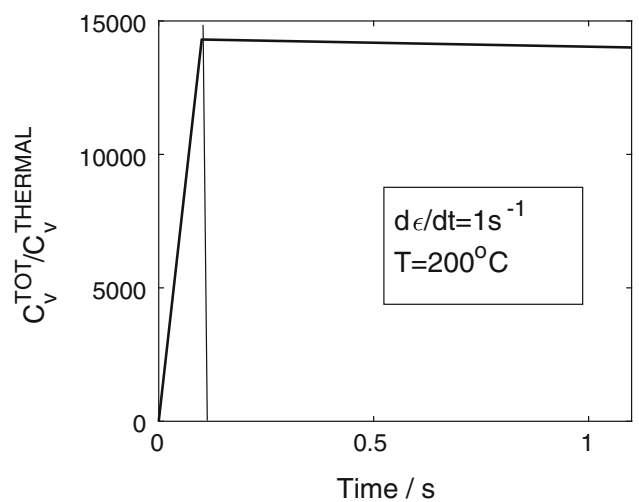

(b)

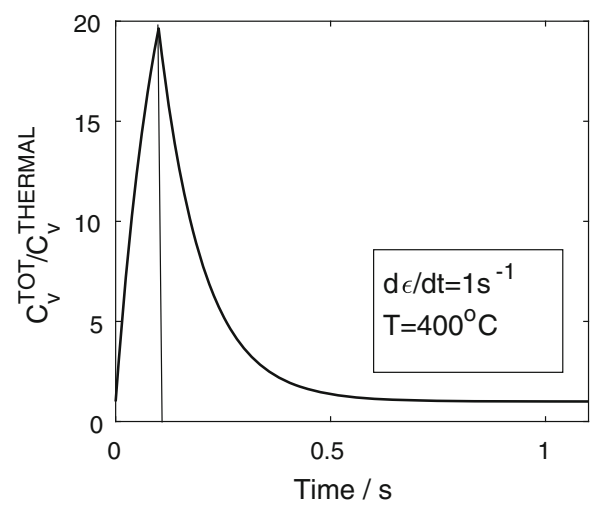

(d)

Fig. 3-Effect on the predicted enhancement in vacancy concentration of increasing strain rate at $473 \mathrm{~K}\left(200{ }^{\circ} \mathrm{C}\right)(a$, b) and increasing temperature $\left[373 \mathrm{~K}\right.$ and $673 \mathrm{~K}\left(100{ }^{\circ} \mathrm{C}\right.$ and $\left.\left.400{ }^{\circ} \mathrm{C}\right)\right]$ at a strain rate of $1 \mathrm{~s}^{-1}(c, d)$. 
vacancies reduces very rapidly with increasing temperature, both during deformation and in the post-deformation decay period.

The effect of strain rate alone (at a constant temperature of $473 \mathrm{~K}\left(200^{\circ} \mathrm{C}\right)$ is considered in Figure 4. In this case, only the excess vacancy concentration during deformation is shown, and the results are plotted as a function of strain rather than time. In the strain rate range shown, $\dot{\epsilon}=10^{-2}$ to $10 \mathrm{~s}^{-1}$, a transition in behaviour as a function of strain is observed. At the lowest strain rate, there is sufficient time for vacancy annihilation to be significant, and a steady-state excess vacancy concentration is reached. For the highest strain rate, the time is sufficiently short that vacancy annihilation is negligible, and the excess vacancy concentration continues to increase linearly with strain.

The key point of these predictions is to demonstrate that a number of different behaviour regimes are possible depending on conditions, lying between two extremes. When vacancy annihilation is negligible (at high values of the Zener Holloman parameter, $Z^{[27]}$ ), the excess vacancy concentration will increase linearly with strain (or time for a constant strain rate). When vacancy annihilation is significant (low $Z$ ) the excess vacancy concentration will reach a steady-state value that depends on strain rate. The typical temperatures and strain rates encountered in testing and processing aluminium alloys encompass both regimes.

Although the focus of this paper is on thermomechanical processing and forming, a brief comment can also be made about creep deformation. Creep is usually characterized by low strain rates, on the order of $10^{-6} \mathrm{~s}^{-1}$, at relatively high homologous temperatures. As discussed in detail elsewhere, ${ }^{[15]}$ in this regime excess vacancies will not accumulate at temperatures above about 0.4 of the melting point $\left(373 \mathrm{~K}, 100{ }^{\circ} \mathrm{C}\right.$ for aluminium). Therefore, in the low strain rate (low stress) creep regime, excess deformation-induced vacancy effects are not expected to be significant. ${ }^{[15]}$

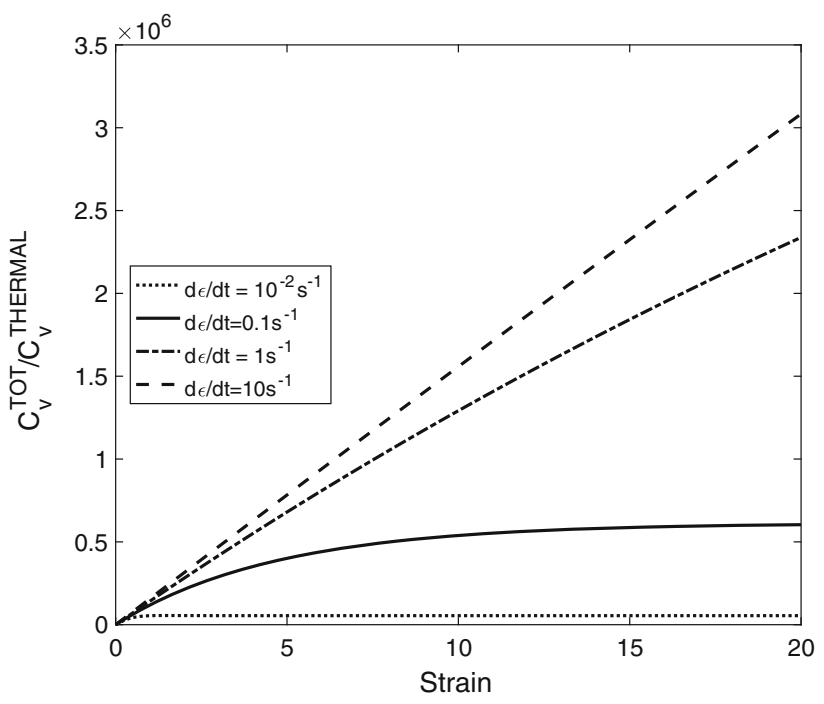

Fig. 4-Effect on the predicted enhancement in vacancy concentration at $473 \mathrm{~K}\left(200{ }^{\circ} \mathrm{C}\right)$ for strain rates from $10^{-2} \mathrm{~s}^{-1}$ to $10 \mathrm{~s}^{-1}$.
This transition in behaviour can be demonstrated on a mechanism map, as shown in Figure 5. This map shows contours for different strain rates that correspond to the point where the vacancy annihilation rate is predicted to reach 1 pct of the vacancy production rate. Regions to the upper right of each contour correspond to conditions where annihilation is "significant", and the opposite is the case towards the lower left. The choice of 1 pct as the boundary between "annihilation significant" and "annihilation insignificant" is arbitrary, but changes in this value result in only small shifts of the contours. The map demonstrates the interplay between strain, strain rate, and temperature in determining whether annihilation is significant or not. For strain rates typical of those in the upper range experienced during industrial thermomechanical processing $\left(1-10 \mathrm{~s}^{-1}\right)$, both high strain and elevated temperature are needed to produce significant annihilation. For the low strain rates and strains typically encountered during tensile testing (e.g. $\dot{\epsilon}=10^{-3} \mathrm{~s}^{-1}$, $\epsilon=10 \mathrm{pct})$, deformation temperatures above $423 \mathrm{~K}$ $\left(150{ }^{\circ} \mathrm{C}\right)$ are predicted to lead to significant annihilation.

\section{Application of Model: Evolving Dislocation Density}

In practice, the dislocation density is not constant but evolves during plastic deformation to reach a steady-state value. As the forest dislocation density increases, the number of vacancy sinks increases. As demonstrated by Militzer et al. ${ }^{[17]}$ this can give behaviour where the excess vacancy concentration reaches a peak with increasing strain before reducing to a steady-state value once the steady-state dislocation density is established. In aluminium, where extensive dynamic recovery occurs even at room temperature, the steady-state dislocation density is established at an applied strain level of approximately 0.3 (the exact value depending on alloy

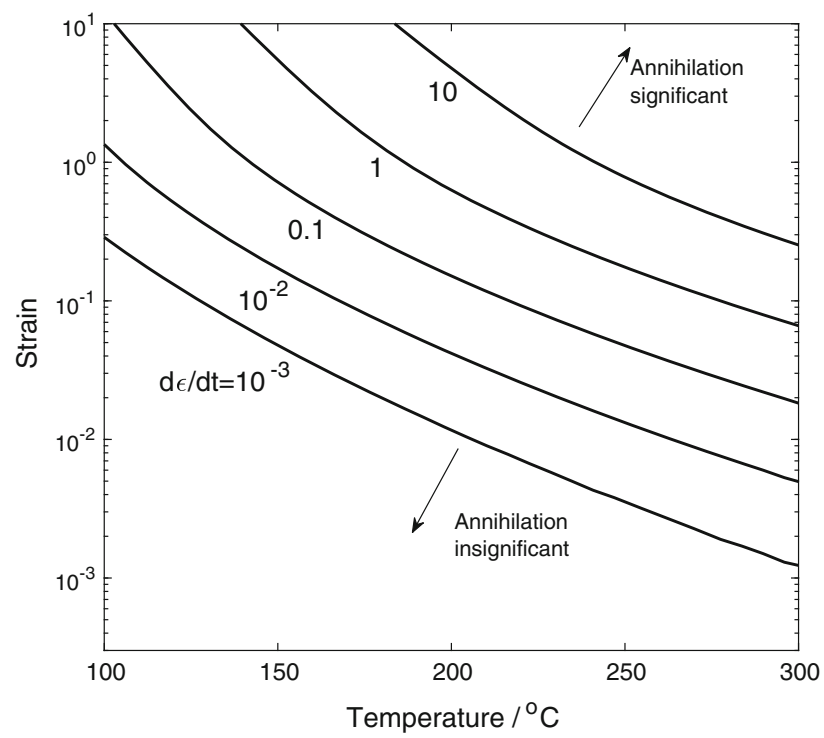

Fig. 5-Mechanism map showing regimes of temperature and strain where vacancy annihilation is predicted to be significant or insignificant during deformation (contours for different strain rates). These regimes give different dependencies on strain and strain rate (see text for details). 
and deformation conditions). ${ }^{[27]}$ Before steady state, the dislocation density increases, and this can be captured in a very simplistic way through Eq. [3]. Figure 6 gives an example comparison of the predicted excess vacancy concentration for the case of evolving and constant dislocation density (the constant dislocation density being fixed at the steady-state value for the evolving dislocation density). The conditions for this simulation are a typical warm forming temperature $\left(473 \mathrm{~K}\left(200{ }^{\circ} \mathrm{C}\right)\right.$ and a strain rate of $10^{-2} \mathrm{~s}^{-1}$ ). A slow strain rate compared to that for industrial forming or thermomechanical processing was chosen since this maximizes the effect of an evolving dislocation density.

When an evolving dislocation density is accounted for, the initial accumulation of excess vacancies is faster because there are fewer dislocation sinks at short times (low strain). For the strain rate and temperature shown, the excess vacancy concentration is then predicted to reach a small peak before decreasing to the steady-state value. This is established at a time of 300 seconds, or a strain of 3 for the simulated strain rate. However, compared with the very large predicted increase in vacancy concentration due to deformation (over four orders of magnitude), the effect of the evolution in dislocation density is small.

A peak in the excess vacancy concentration is observed if the point at which the vacancy annihilation rate balances the vacancy production rate occurs whilst the dislocation density is still increasing. This means the number of sinks for excess vacancies is increasing, which then leads to an annihilation rate that exceeds the production rate, causing a decrease in the number of excess vacancies until the steady state is established. Temperature and strain rate will influence the relative difference between the point at which the steady-state dislocation density is established and the point at which vacancy annihilation matches production. For example, Figure 7 shows the effect of temperature on the predicted occurrence of a peak in the excess vacancy concentration (for a strain rate of $10^{-2} \mathrm{~s}^{-1}$ ). On this plot,

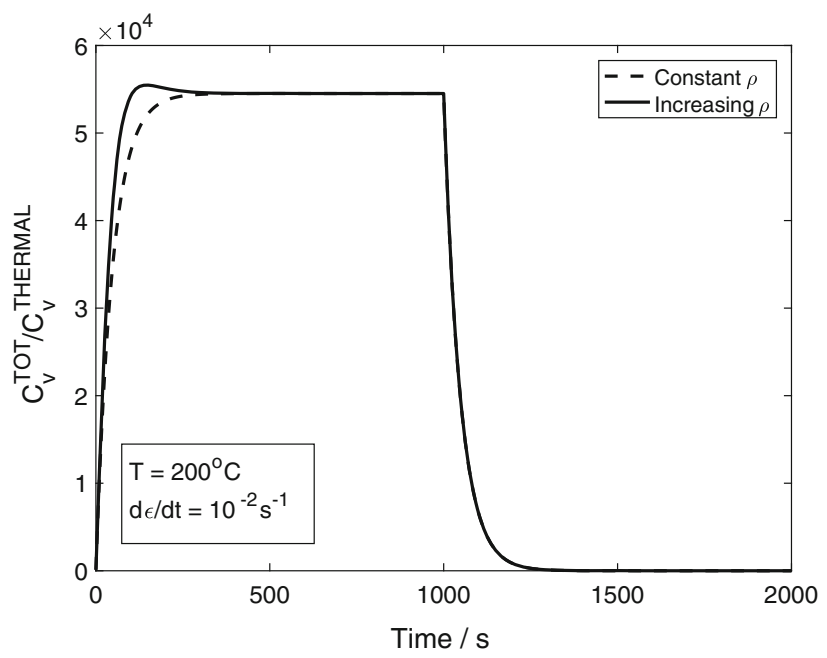

Fig. 6-A comparison of predicted enhancement in vacancy concentration at $473 \mathrm{~K}\left(200{ }^{\circ} \mathrm{C}\right), \dot{\epsilon}=10^{-2} \mathrm{~s}^{-1}$ assuming either a constant dislocation density or a dislocation density increasing to the constant value. the excess vacancy ratios have been normalized (to the maximum value at each temperature), allowing the peak shapes to be directly compared. As already demonstrated, vacancy annihilation is greatly enhanced at increasing temperature, and this effect is stronger than the reduction in time required to reach the steady-state dislocation density. Therefore, as the temperature increases, a more pronounced peak is observed. The peak disappears at lower temperature where a constant dislocation density becomes established before vacancy annihilation becomes significant.

The role of vacancy annihilation and the effect of temperature and strain rate on its importance can be more clearly shown by plotting the ratio of the vacancies annihilated to those created. An example of such a plot for a range of deformation conditions is shown in Figure 8, allowing for an evolving dislocation density.

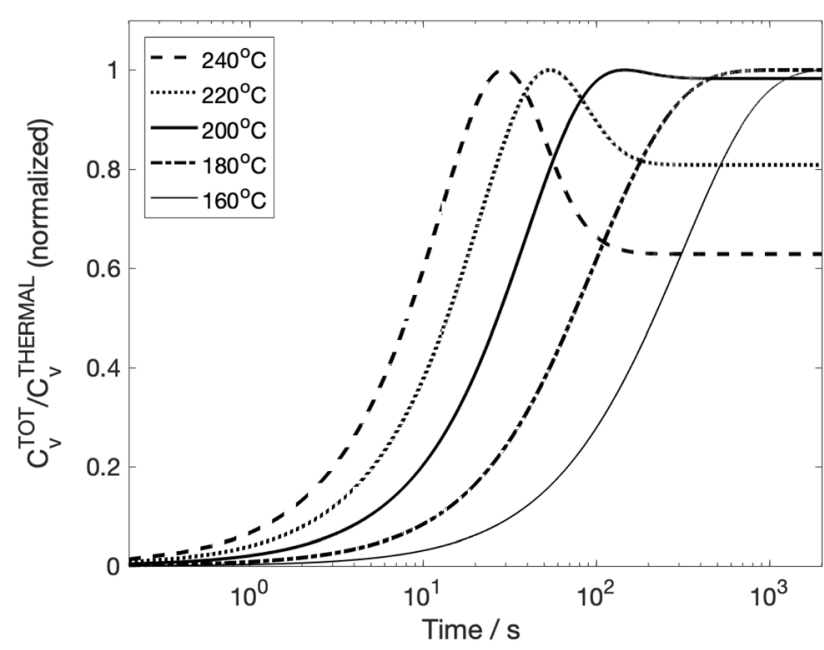

Fig. 7-Predicted effect of an increase in temperature on the enhancement in vacancy concentration (normalized) for increasing temperatures in the range $433 \mathrm{~K}$ to $513 \mathrm{~K}\left(160{ }^{\circ} \mathrm{C}\right.$ to $\left.240{ }^{\circ} \mathrm{C}\right)$, $\dot{\epsilon}=10^{-2} \mathrm{~s}^{-1}$.

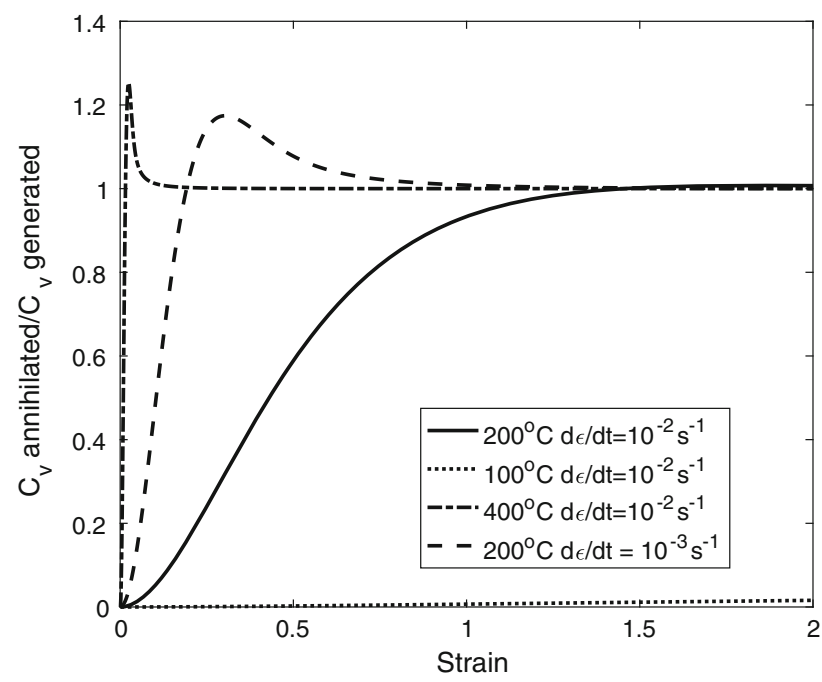

Fig. 8-Predicted ratio of vacancies annihilated to vacancies generated for a range of conditions of temperature and strain rate (see legend). 
Strain is plotted as the abscissa rather than time to enable the effect of changes in strain rate and temperature to be compared at iso-strain. A steady-state excess vacancy concentration is established when the ratio of annihilated to created vacancies is 1 . Values greater than this correspond to more vacancies being annihilated than created and values less correspond to more vacancies being created than annihilated. As already discussed, for a given strain rate $\left(\right.$ e.g. $\left.10^{-2} \mathrm{~s}^{-1}\right)$, the vacancy annihilation rate increases strongly with temperature, and at high temperature, steady state is achieved at relatively low strain $(\simeq 10 \mathrm{pct})$. On the other hand, for the same strain rate but with a deformation temperature of $373 \mathrm{~K}\left(100{ }^{\circ} \mathrm{C}\right)$, vacancy annihilation is almost negligible, even up to a strain of 2 .

\section{E. Application of Model: Vacancy Production and Annihilation Parameters}

A key factor limiting the accuracy of the excess vacancy model is the uncertainty in some of the important parameters. The effect of small changes in the activation energy for vacancy diffusion has already been discussed, but there are two other parameters in Eq. [1] that are also important and are poorly defined. The first is $\chi$, which is the fraction of the applied work that is used to produce excess vacancies from mechanical jogs. Estimates for this parameter from experiment range greatly, from around 2 to 10 pct. ${ }^{[13,17]}$ It is estimated that around 30 pct of the total stored energy after cold deformation is associated with excess vacancies (in $\mathrm{Cu}$ ) ${ }^{[16]}$ but this is hard to measure since the excess vacancies anneal rapidly above cryogenic temperatures. Furthermore, it is common to assume $\chi$ is independent of temperature, strain and strain rate, but in practice this is likely to not be the case. This point will be returned to later.

The $\chi$ parameter controls the number of excess vacancies that are created for a given increment in strain. The effect of changing this parameter are shown in Figure 9(a) for an example temperature of $473 \mathrm{~K}$ $\left(200{ }^{\circ} \mathrm{C}\right.$ ) and a strain rate of $10^{-2} \mathrm{~s}^{-1}$. Deformation is applied for 1000 seconds and then excess vacancy decay is predicted for a further 1000 seconds. Note that the highest $\chi$ value shown here is not expected to be physically reasonable, but is plotted to demonstrate the functional dependence of the excess vacancies when $\chi$ is doubled. As expected from inspection of Eq. [1], when mechanical jogs dominate, doubling $\chi$ leads to a doubling in the rate of excess vacancy accumulation and a doubling of the steady-state vacancy concentration. The excess vacancy decay rate is not affected by changes to $\chi$ since this only influences vacancy production.

The other uncertain parameter is $\kappa$. This is the dislocation arrangement term, and is used to capture the effect that the inhomogeneity in dislocation distribution will have on the diffusion distance for excess

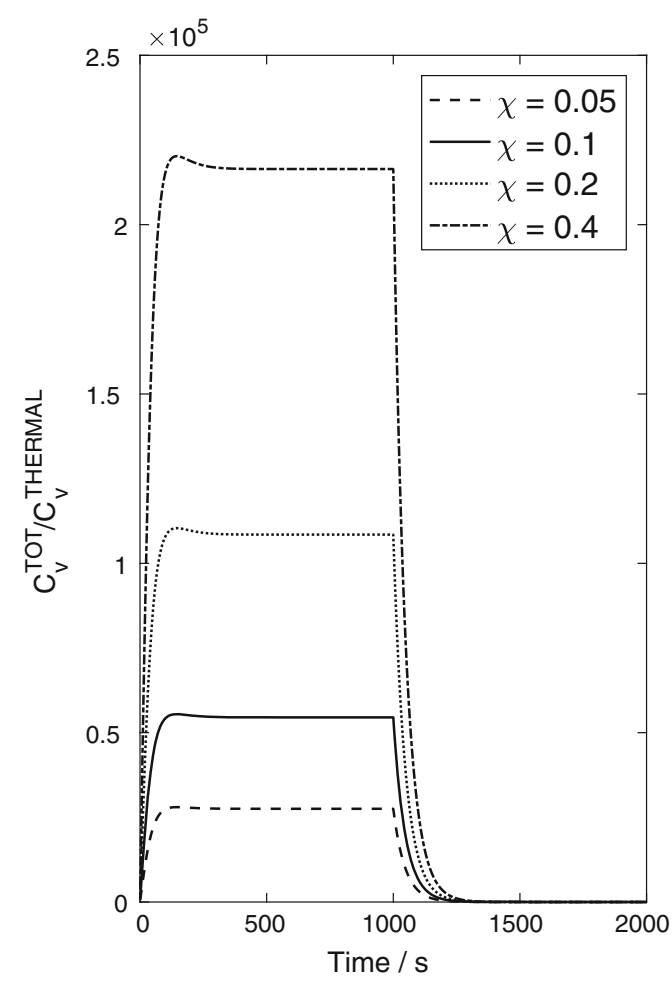

(a)

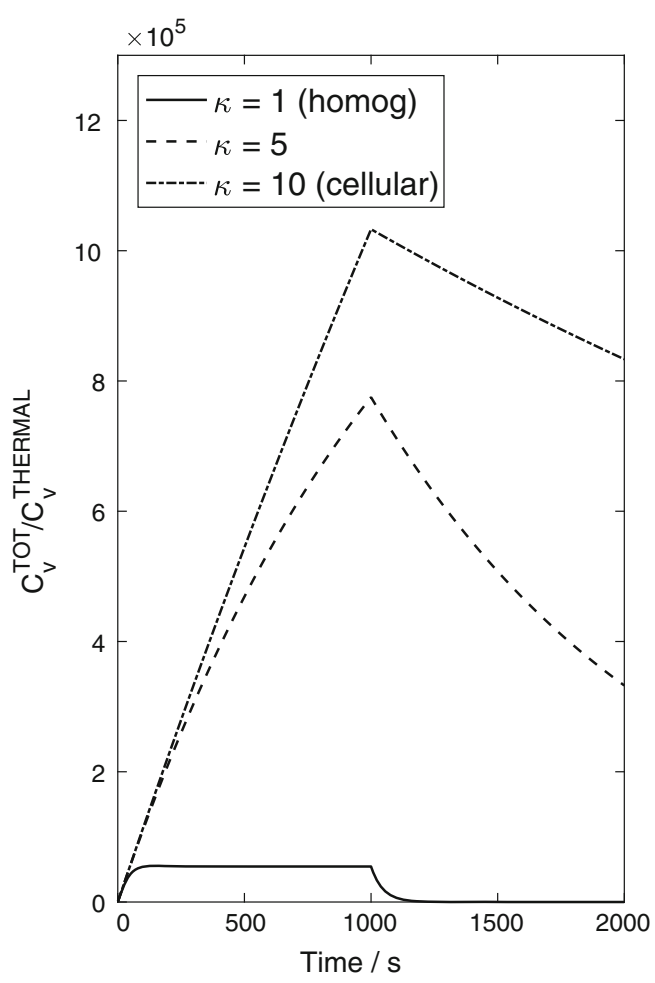

(b)

Fig. 9-Predicted enhancement in vacancy concentration at $473 \mathrm{~K}\left(200{ }^{\circ} \mathrm{C}\right), \dot{\epsilon}=10^{-2} \mathrm{~s}^{-1}$ for variations in $(a) \chi$, the fraction of the applied work consumed in producing vacancies, $(b) \kappa$ the dislocation arrangement parameter ( $\kappa=1$ homogeneous, $\kappa=10$ cellular). 
vacancies to migrate to sinks. The value is usually in the range from 1 for a homogeneous distribution of dislocations to approximately 10 for a fully cellular arrangement. $^{[17]}$ The correct value to chose for this parameter is complicated by the fact that the dislocation arrangement itself is changing during deformation. If dislocation rearrangement is much slower than excess vacancy production and annihilation, then assuming $\kappa \simeq 1$ may be reasonable.

The effect of changing $\kappa$ on the predictions for a temperature of $473 \mathrm{~K}\left(200{ }^{\circ} \mathrm{C}\right)$ and a strain rate of $10^{-2} \mathrm{~s}^{-1}$ is shown in Figure 9(b). Changing $\kappa$ influences both the accumulation of excess vacancies during the deformation phase and the rate of recovery once deformation finishes. A larger value of $\kappa$ results in a reduced rate of vacancy annihilation. This leads to a longer time (higher strain) to reach steady state, a higher excess vacancy concentration, and a longer annihilation time after deformation.

\section{F. Application of Model: Effect on Diffusivity}

One of the most important effects of excess vacancies is in enhancing mass transport through an increase in diffusivity. The major solutes used in aluminium alloys

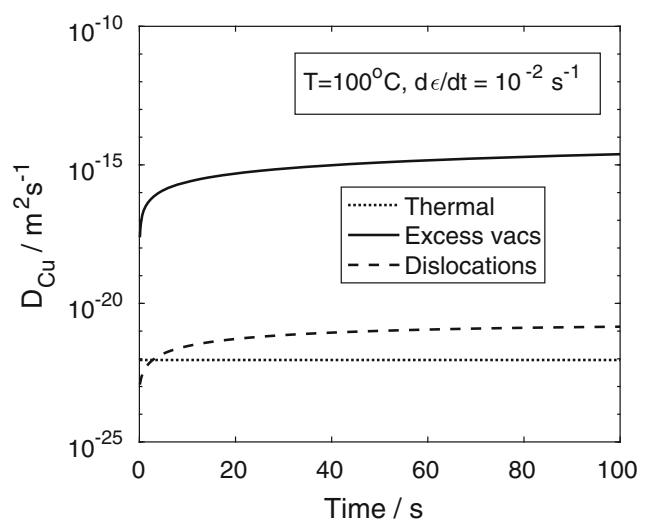

(a)

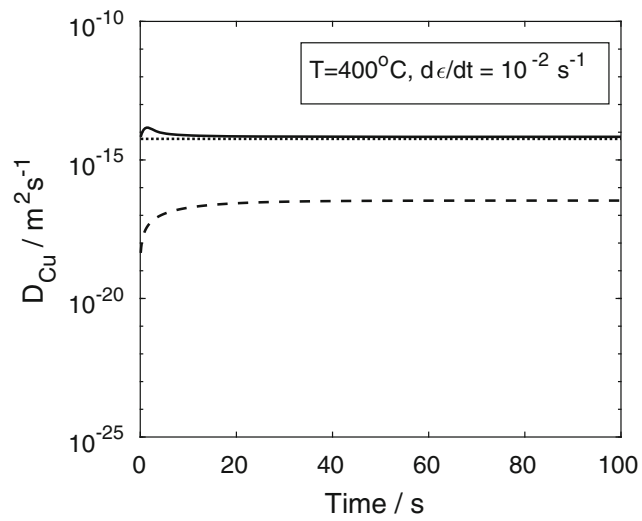

(c) are substitutional in nature and require vacancies to jump. In this case, the diffusivity increases in proportion to the excess vacancy concentration, as shown in Eq. [5]. The dislocations induced by deformation also directly enhance diffusivity though acting as fast diffusion pipes. This is accounted for in Eq. [5]. To help understand the relative contribution of these effects, the excess vacancy and dislocation contributions to Eq. [5] have been calculated separately and are shown in Figure 10 for a range of conditions. These calculations were performed assuming $\mathrm{Cu}$ as the solute species since this is one of the slowest diffusing precipitate forming alloying additions widely used in aluminium alloys. The necessary $D_{\mathrm{s} 0}$ and $Q_{\text {s }}$ values are reported in Table I.

Figures 5(a) through (c) show the effect of increasing temperature $373 \mathrm{~K}$ to $673 \mathrm{~K}\left(100{ }^{\circ} \mathrm{C}\right.$ to $\left.400{ }^{\circ} \mathrm{C}\right)$ for a fixed strain rate $\left(\dot{\epsilon}=10^{-2} \mathrm{~s}^{-1}\right)$. Figures 5(a) through (c) shows the effect of an increased strain rate $\left(\dot{\epsilon}=1 \mathrm{~s}^{-1}\right)$ at $473 \mathrm{~K}\left(200{ }^{\circ} \mathrm{C}\right)$. In all cases, the total deformation strain was 1 . The critical point demonstrated by Figure 5 is that for the wide range of temperatures shown, the enhancement of diffusivity due to excess vacancies is always predicted to be several orders of magnitude greater than that due to dislocations. As expected, the effect of defects (dislocations and excess vacancies) in

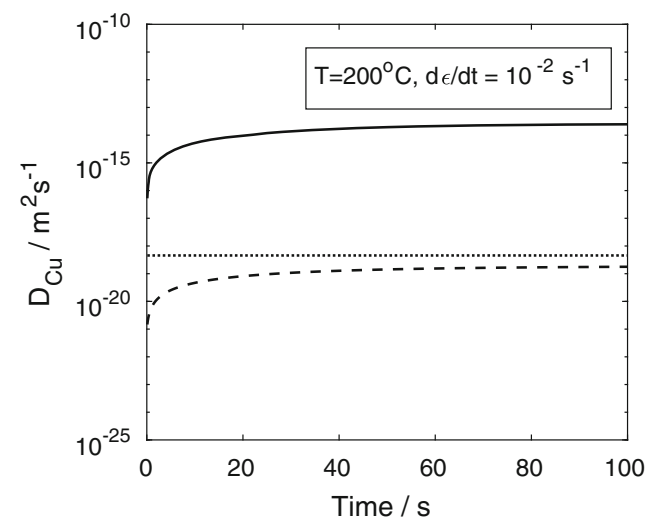

(b)

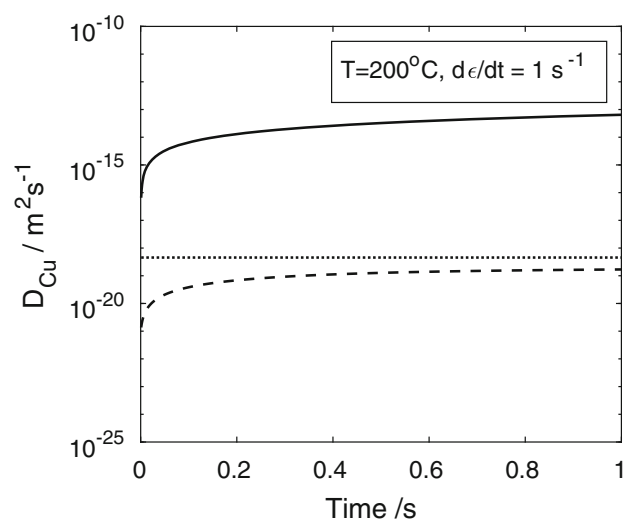

(d)

Fig. 10-Predicted diffusivity of $\mathrm{Cu}$ in aluminium comparing thermal effects alone with the contribution from diffusion along grain boundaries and due to a deformation enhanced vacancy concentration. (a) $T=373 \mathrm{~K}\left(100{ }^{\circ} \mathrm{C}\right), \dot{\epsilon}=10^{-2} \mathrm{~s}^{-1},(b) T=473 \mathrm{~K}\left(200{ }^{\circ} \mathrm{C}\right), \dot{\epsilon}=10^{-2} \mathrm{~s}^{-1},(c) T=$ $673 \mathrm{~K}\left(400{ }^{\circ} \mathrm{C}\right), \dot{\epsilon}=10^{-2} \mathrm{~s}^{-1},(d) T=473 \mathrm{~K}\left(200{ }^{\circ} \mathrm{C}\right), \dot{\epsilon}=1 \mathrm{~s}^{-1}$. 
enhancing diffusivity is stronger at lower temperature and higher strain rate. However, even at $673 \mathrm{~K}\left(400{ }^{\circ} \mathrm{C}\right)$, $\dot{\epsilon}=10^{-2} \mathrm{~s}^{-1}$, the excess vacancies are predicted to lead to a small enhancement in diffusivity whilst the dislocation enhancement at this temperature and strain rate is negligible. At $473 \mathrm{~K}\left(200{ }^{\circ} \mathrm{C}\right) \dot{\epsilon}=10^{-2} \mathrm{~s}^{-1}$, the excess vacancies are predicted to lead to an increase in diffusivity that is five orders of magnitude or more above that expected from thermal vacancies alone.

\section{EXTENDING THE MODEL}

The classical excess vacancy model used here (and expressed in Eq. [1]) ignores some physical effects that can be important in aluminium alloys, depending on the deformation conditions and alloy microstructure. The first is the effect of strain localization during plastic deformation. Implicit in the previous discussion is the assumption that the distribution of excess vacancies is homogeneous. This will be reasonable in the case where the sources of the deformation-induced vacancies are closely spaced compared to the mean vacancy diffusion distance. This is true when the slip band spacing is small compared to the vacancy diffusion distance. ${ }^{[15]}$ However, the other limiting case occurs when deformation occurs heterogeneously and is concentrated in slip bands whose spacing is large compared to the vacancy diffusion distance. In this case, a heterogeneous distribution of excess vacancies will arise, and futhermore the excess vacancy concentration will be different to that in the homogeneous case. The two extreme cases have been considered by Mecking and Estrin, ${ }^{[15]}$ who showed that in the case of localized widely spaced bands, there is a net increase in the excess vacancy concentration compared to the homogeneous case. The amplification factor in the excess vacancy concentration caused by strain localization can be several orders of magnitude.

Using the assumption that slip is confined to bands that are very narrow compared to their spacing, the concentration profile of excess vacancies can be estimated perpendicular to the band (as excess vacancies migrate from the slip band into the surrounding material) ${ }^{[15]}$ As shown by Mecking and Estrin, ${ }^{[15]}$ the vacancy concentration profile reaches a steady state whose width depends only on the distance between excess vacancy sinks. Figure 11 shows the predicted vacancy concentration profile at steady state around a line source, estimating the annihilation distance from the steady-state dislocation density (Table I).

Comparing the distance over which the vacancy concentration decays with the expected slip spacing will determine whether homogeneous or heterogeneous excess vacancy behaviour is expected. For pure aluminium, high stacking fault energy disfavours localization and the slip spacing is expected to be orders of magnitude less than the width of the concentration profile. ${ }^{[28,29]}$ In this case the assumption of a homogeneous excess vacancy distribution is reasonable.

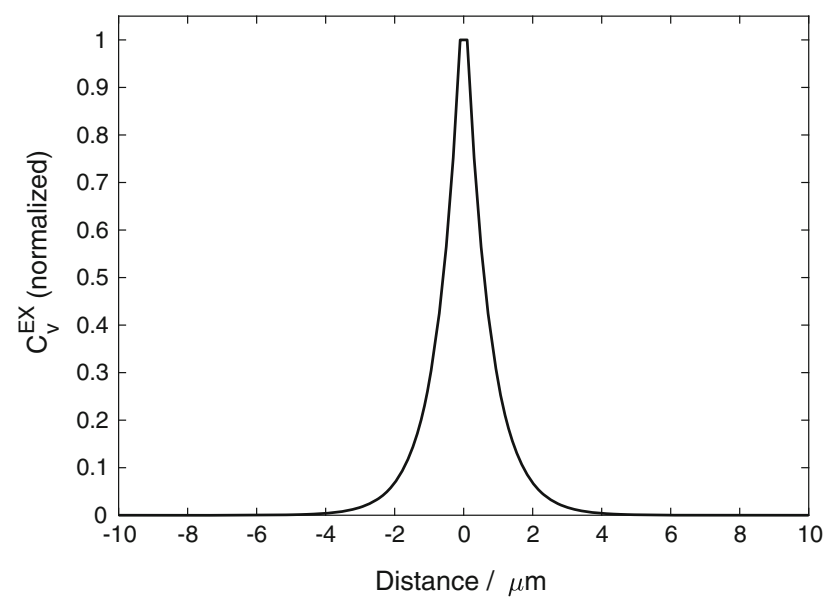

Fig. 11-Steady-state (normalized) excess vacancy concentration profile around a source for an assumed sink spacing corresponding to a dislocation density $\rho=2 \times 10^{12} \mathrm{~m}^{-2}$.

However, many aluminium alloys contain shearable precipitates that can lead to strain localization and more widely spaced slip bands. ${ }^{[30,31]}$ These bands can be several $\mu \mathrm{m}$ apart depending on alloy and precipitate state. ${ }^{[32-34]}$ At this spacing, the excess vacancy distribution would be expected to remain heterogenous, with only partial (or no) overlap between the volumes containing excess vacancies originating from each slip band. In this case, the excess vacancies would be expected to have a heterogeneous effect on the microstructure since they would have a stronger effect on diffusivity within the slip bands than between them. Properly accounting for this effect requires a more physically realistic deformation simulation in which strain localisation is considered.

The second physical effect that is ignored in the Militzer et al. excess vacancy model is related to the vacancy annihilation process. In Eq. [1], two annihilation sites are considered, forest dislocations (assumed to be static) and grain boundaries. Unless the grains are ultra-fine, the forest dislocation sinks dominate the annihilation process. These annihilation processes are diffusion controlled and depend on temperature but not strain or strain rate.

However, another annihilation mechanism is possible, involving sweeping of excess vacancies by mobile dislocations. Atomistic simulations (in $\mathrm{Cu}$ ) suggest that vacancies within about two atomic distances of the dislocation core (or jog-line) have much lower energy barriers for movement compared to the bulk. ${ }^{[35]}$ Vacancies within this distance of moving dislocation could therefore be swept up by moving jogged dislocations, with some of them becoming annihilated at the jogs, which act as vacancy sinks. An accurate calculation of this effect is complex because it depends on the relative velocity of the dislocation compared to the vacancy jump frequency, the spacing of jogs along the dislocation line, the distribution of the vacancies, and the homogeneity of the mobile dislocations. Nevertheless, 
an upper bound estimate of the significance of this effect can be made by calculating the total volume within the assumed capture distance of mobile dislocations (2 atomic distances above and below the line, giving a capture width of $4 b$ ). The Orowan law relates the increment in (shear) strain rate to the average mobile dislocation velocity $v$ :

$$
\dot{\epsilon}=\rho_{\mathrm{m}} b v .
$$

Assuming a homogeneous distribution of mobile dislocations, and ignoring overlap between swept volumes, the increment of volume fraction within the capture volume of mobile dislocations in a time interval $\mathrm{d} t$, assuming a swept width of $4 b$, is given by:

$$
\mathrm{d} V_{\mathrm{s}}=4 b \rho_{\mathrm{m}} v \mathrm{~d} t
$$

Combining Eqs. [7] and [8] gives a simple relationship between the rate of increase in the capture volume and the shear strain rate:

$$
\frac{\mathrm{d} V_{\mathrm{s}}}{\mathrm{d} t}=4 \dot{\epsilon}
$$

This analysis is a gross approximation to the real physical processes occurring, and thus is not sufficiently reliable for quantitative calculations. Nevertheless, the important point is that as the strain rate increases, the generation of more excess vacancies (per unit time) will be countered by more of those vacancies being captured by other moving dislocations. Using this simple upper bound calculation, a shear strain of 0.25 of greater would correspond to the entire volume being within the capture range of mobile dislocations.

One implication of this mechanism is that the excess vacancy concentration will not continue to increase linearly with strain, even in the absence of diffusion of vacancies to forest dislocations (e.g. at low temperature). Instead, a steady state will eventually become established where the generation of excess vacancies will be balanced by their absorption by mobile dislocations. An important consideration is the lifetime of an excess vacancy before it is annihilated, either by diffusion to a forest dislocation or capture by a moving dislocation. If the vacancy survives for long enough to transport solute over the distances required to form a precipitate, strong enhancement of precipitate nucleation and growth will occur, regardless of whether than vacancy is eventually annihilated. The vacancy lifetime is thus an important characteristic, which will be related to the temperature in the case of diffusion to forest dislocations and strain rate (i.e. dislocation velocity) in the case of mobile dislocation capture.

\section{COMPARISON WITH QUENCHING}

In aluminium alloys, another important source of excess vacancies can arise from rapid quenching. It is well known that the elevated vacancy concentration retained on quenching has a strong effect on diffusion at low temperature, facilitating processes such as formation of GP zones (natural ageing) ${ }^{[36]}$ It is interesting to compare the excess vacancy concentrations that arise from the quenching process with those predicted due to deformation. For simplicity, solute effects ${ }^{[37]}$ will be ignored and a perfect quench will be assumed, in which all the vacancies from high temperature are retained. Figure 12(a) shows a comparison of the quench-induced and strain-induced excess vacancy concentrations as a function of initial temperature and strain respectively. It can be seen that for room temperature deformation or high strain rate/high-temperature deformation typical of thermomechanical processing operations $(673 \mathrm{~K}$ $\left.\left(400{ }^{\circ} \mathrm{C}\right), \dot{\epsilon}=1 \mathrm{~s}^{-1}\right)$ it is predicted that the deformation-induced vacancy concentration can exceed the quench-induced vacancy concentration by an order of magnitude, but is always within 2 orders of magnitude for the range of conditions shown here. For warm deformation at a typical laboratory tensile test strain rate $\left(523 \mathrm{~K}\left(250{ }^{\circ} \mathrm{C}\right), \dot{\epsilon}=10^{-3} \mathrm{~s}^{-1}\right)$, the excess vacancy concentrations due to quenching and deformation are of similar magnitude (given the large uncertainty arising from the simplicity of both models). Overall, the results show that both quenching and deformation can lead to comparable increases in vacancy concentration of many orders of magnitude greater than the equilibrium room temperature value $\left(\simeq 2 \times 10^{13} \mathrm{~s}^{-1}\right)$.

Another important characteristic of the excess vacancies is the duration they persist after deformation or quenching. If the vacancies annihilate very quickly, then their opportunity to affect nucleation and diffusion processes will be limited. There is an important difference here between deformation and quench-induced excess vacancies. During deformation, there is a relatively high dislocation density, and therefore a large number of excess vacancy sinks. The dislocation density in undeformed aluminium is approximately $1 \times 10^{11} \mathrm{~m}^{-2} \cdot{ }^{[38]}$ This is about two orders of magnitude less than the steady state achieved during deformation. ${ }^{[39]}$ The effect of this on the time to annihilate the excess vacancies due to quenching and strain respectively is shown in Figure 12(b). For this calculation, it was assumed the dislocations after quenching have a cellular arrangement, $\kappa=10$. The annealing temperature prior to the quench was $873 \mathrm{~K}\left(600{ }^{\circ} \mathrm{C}\right)$ and the temperature after the quench was $423 \mathrm{~K}\left(150^{\circ} \mathrm{C}\right)$. For deformation, the temperature was $423 \mathrm{~K}\left(150^{\circ} \mathrm{C}\right)$ and the strain/strain rate were 0.1 and $10^{-3} \mathrm{~s}^{-1}$ respectively. These conditions were chosen to compare with a more sophisticated calculation of vacancy annihilation after quenching in aluminium reported in the literature. ${ }^{[40]}$ Although the absolute values of time are not expected to be accurate, the important point that the excess vacancies produced by deformation will annihilate much faster than those produced after quenching is well demonstrated. For the conditions show here, there is about a two order of magnitude difference between the predicted annihilation times. A more sophisticated vacancy annihilation model of Fisher et al. ${ }^{40]}$ gives a 

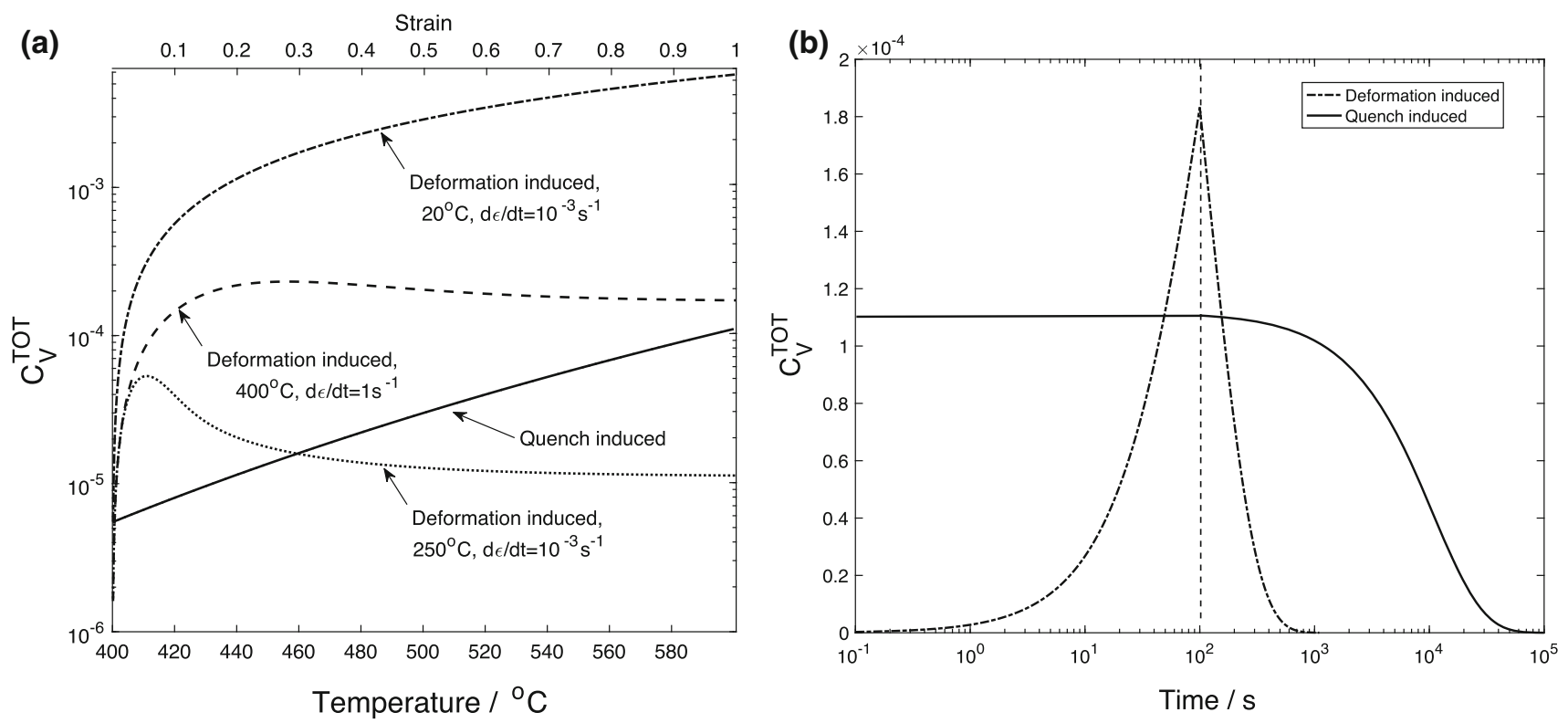

Fig. 12-(a) A comparison of the predicted vacancy concentration due to deformation (as a function of strain) and trapped by quenching from elevated temperature (as a function of initial temperature). (b) A comparison of vacancy accumulation and decay for deformation and quench-induced excess vacancies $\left(t=10^{2} \mathrm{~s}\right.$ at deformation stop or instantaneous quench respectively). For deformation, $T=423 \mathrm{~K}, 150{ }^{\circ} \mathrm{C}$, $\dot{\epsilon}=10^{-3} \mathrm{~s}^{-1}, \epsilon=0.1$. For quenching; initial temperature $\left.873 \mathrm{~K}\left(600{ }^{\circ} \mathrm{C}\right)\right)$. After deformation or quench $T=423 \mathrm{~K}, 150^{\circ} \mathrm{C}$.

similar difference in order of magnitude between annihilation times for the dislocation densities assumed here, but both times are much shorter. It may be concluded that whilst the excess vacancies produced by quenching persist for long times (hours) after the quench and can thus influence precipitation kinetics over this period, the comparable number of excess vacancies produced by deformation are rapidly annihilated (in seconds ${ }^{[40]}$ ) and thus it is their action during deformation that is most important.

\section{CONCLUSIONS}

Deformation-induced excess vacancies can have a strong effect in enhancing diffusion kinetics in aluminium alloys, greatly accelerating processes such as precipitate nucleation, growth, and coarsening. The application and implications of a simple model to predict deformation-induced excess vacancies when applied to typical conditions used for testing and thermomechanical processing of aluminium alloys have been investigated. The following conclusions can be drawn from this work:

1. The excess vacancy concentration produced by deformation is predicted to lead to increases in diffusivity that can be more than five orders of magnitude greater than that without deformation. The enhancing effect falls rapidly with increasing temperature and is negligible above around $673 \mathrm{~K}$ $\left(400{ }^{\circ} \mathrm{C}\right)$.

2. The conditions used for processing and testing aluminium alloys encompass two distinct regimes with regard to the balance between excess vacancy production and annihilation. At low temperatures or high strain rates (high $Z$ ), vacancy annihilation is suppressed compared to production and the excess vacancy concentration increases with strain but is insensitive to strain rate. At high temperatures or low strain rates (low $Z$ ) vacancy annihilation and production will rapidly reach a steady-state concentration, which increases with strain rate but is insensitive to strain.

3. The quantitative accuracy of the model is limited by the uncertainty in key input parameters such as vacancy migration energy, dislocation arrangement, and fraction of the applied work used to produce point defects. A pragmatic method is presented for calibrating the parameters that control vacancy annihilation based on in situ measurement of the decay of precipitate growth rate after deformation is complete.

4. The excess vacancy concentrations produced by deformation can be comparable to those produced by rapid quenching from temperatures close to the melting point. A key difference is that the annihilation of deformation-induced excess vacancies is predicted to be two orders of magnitude faster than those due to quenching due to the greater number of vacancy sinks in the former case.

5. To improve the quantitative accuracy of the present model requires a more realistic dislocation model that properly considers the evolving dislocation distribution, a better estimate of vacancy annihilation by mobile dislocations, and a more accurate determination of key input parameters such as the activation energy for vacancy diffusion. The challenge is to improve the model without requiring a full dislocation dynamics simulation, which is computationally expensive. 


\section{ACKNOWLEDGMENTS}

The EPSRC are thanked for financial support through the associated programme grant LightFORM (EP/R001715/1). The author is grateful to Prof Chris Hutchinson (Monash University) for valuable discussions on the topic of this paper. The data required to reproduce these findings are available from the LightFORM Zenodo repository https://zenodo.org/commu nities/lightform/.

\section{OPEN ACCESS}

This article is licensed under a Creative Commons Attribution 4.0 International License, which permits use, sharing, adaptation, distribution and reproduction in any medium or format, as long as you give appropriate credit to the original author(s) and the source, provide a link to the Creative Commons licence, and indicate if changes were made. The images or other third party material in this article are included in the article's Creative Commons licence, unless indicated otherwise in a credit line to the material. If material is not included in the article's Creative Commons licence and your intended use is not permitted by statutory regulation or exceeds the permitted use, you will need to obtain permission directly from the copyright holder. To view a copy of this licence, visit http://creat ivecommons.org/licenses/by/4.0/.

\section{REFERENCES}

1. A. Deschamps, F. Bley, F. Livet, D. Fabregue, and L. David: Philos. Mag., 2003, vol. 83, pp. 677-92.

2. A. Deschamps, G. Fribourg, Y. Bréchet, J. Chemin, and C. Hutchinson: Acta Mater., 2012, vol. 60, pp. 1905-16.

3. C. Hutchinson, F. de Geuser, Y. Chen, and A. Deschamps: Acta Mater., 2014, vol. 74, pp. 96-109.

4. M. Starink, N. Gao, N. Kamp, S. Wang, P. Pitcher, and I. Sinclair: Mater. Sci. Eng.: A, 2006, vol. 418, pp. 241-49.

5. W. Sun, Y. Zhu, R. Marceau, L. Wang, Q. Zhang, X. Gao, and C. Hutchinson: Science, 2019, vol. 363, pp. 972-75.

6. Y. Zhang, S. Jin, P. Trimby, X. Liao, M. Murashkin, R. Valiev, J. Liu, J. Cairney, S. Ringer, and G. Sha: Acta Mater., 2019, vol. 162, pp. 19-32.

7. J.D. Embury, A. Deschamps, and Y. Brechet: Scripta Mater., 2003, vol. 49, pp. 927-32.

8. A. Deschamps, F. Bley, F. Livet, and D. Fabregue: Philos. Mag. A, 1999, vol. 79, pp. 2485-2504.

9. Y. Brechet and Y. Estrin: Acta Metall. Mater., 1995, vol. 43, pp. $955-63$.
10. L. Couturier, A. Deschamps, F. De Geuser, F. Fazeli, and W. Poole: Scripta Mater., 2017, vol. 136, pp. 120-23.

11. G. Saada: Physica, 1961, vol. 27, pp. 657-60.

12. G. Saada: Acta Metall., 1961, vol. 9, pp. 166-68.

13. F. Seitz: Adv. Phys., 1952, vol. 1, pp. 43-90.

14. A. Seeger, The London, Edinburgh, and Dublin Philosophical Magazine and Journal of Science, 1955, vol. 46, pp. 1194-1215.

15. H. Mecking and Y. Estrin: Scripta Metall., 1980, vol. 14, pp. 815-19.

16. G. Gottstein, J. Bewerunge, H. Mecking, and H. Wollenberger: Acta Metall., 1975, vol. 23, pp. 641-52.

17. M. Militzer, W.P. Sun, and J.J. Jonas: Acta Metall. Mater., 1994, vol. 42 , pp. $133-41$.

18. S. Khani Moghanaki and M. Kazeminezhad, J. Alloys Compd., 2016 vol. 683 , pp. $527-32$.

19. K.E. Tello, A.P. Gerlich, and P.F. Mendez: Sci. Technol. Weld. Join., 2010, vol. 15 , pp. 260-66.

20. J.W. Christian, The Theory of Transformations in Metals and Alloys, 2002, Newnes, pp. 416.

21. G.E. Totten and D.S. MacKenzie, Handbook of Aluminum, Vol. 1, CRC Press, 2003, pp. 83

22. J. Embury and R. Nicholson: Acta Metall., 1965, vol. 13, pp. 403-17.

23. Y. Du, Y.A. Chang B. Huang, W. Gong, Z. Jin, H. Xu, Z. Yuan, Y. Liu, Y. He, and F.-Y. Xie, Mater. Sci. Eng: A, 2003, vol. 363, pp. $140-51$.

24. R. Ferragut, A. Dupasquier, C. Macchi, A. Somoza, R. Lumley, and I. Polmear: Scripta Mater., 2009, vol. 60, pp. 137-40.

25. A. Dupasquier, G. Kögel, and A. Somoza: Acta Mater., 2004, vol. 52, pp. 4707-26.

26. T.W. Gullion and M.S. Conradi: Phys. Rev. B, 1987, vol. 36, pp. $3880-83$.

27. F.J. Humphreys and M. Hatherley, Recrystallization and Related Annealing Phenomena, 2nd Edition, Elsevier, 2004, p. 18.

28. D. Kuhlmann-Wilsdorf, J. van der Merwe, and H. Wilsdorf, The London, Edinburgh, and Dublin Philosophical Magazine and Journal of Science, 1952, vol. 43, pp. 632-44.

29. E. Hornbogen and E. Starke: Acta Metall. Mater., 1991, vol. 41, pp. $1-16$

30. G.M. Ludtka and D.E. Laughlin: Metall. Trans. A., 1981, vol. 1981 (12), pp. 2083-91.

31. A. Luft: Prog. Mater. Sci., 1991, vol. 35, pp. 97-204.

32. T. Dorin, F. De Geuser, W. Lefebvre, C. Sigli, and A. Deschamps: Mater. Sci. Eng.: A, 2014, vol. 605, pp. 119-26.

33. T. Sanders, Jr and E.A. Starke, Jr: Acta Metall., 1982, vol. 30, pp. 927-39.

34. K.V. Jata and E.A. Starke: Metall. Trans. A, 1986, vol. 17, pp. 1011-26.

35. T. Vegge and K.W. Jacobsen: J. Phys., 2002, vol. 14, pp. 2929-56.

36. D. Turnbull and R. Cormia: Acta Metall., 1960, vol. 8, pp. 747-50.

37. K.H. Westmacott, R.S. Barnes, D. Hull, and R.E. Smallman: Philos. Mag., 1961, vol. 6, pp. 929-35.

38. G.K. Williamson and R.E. Smallman: Philos. Mag., 1956, vol. 1, pp. $34-46$.

39. C.M. Sellars and Q. Zhu: Mater. Sci. Eng.: A, 2000, vol. 280, pp. 1-7.

40. F. Fischer, J. Svoboda, F. Appel, and E. Kozeschnik: Acta Mater., 2011, vol. 59, pp. 3463-72.

Publisher's Note Springer Nature remains neutral with regard to jurisdictional claims in published maps and institutional affiliations. 